\title{
A message for 2020
}

\author{
Naomichi Matsumoto ${ }^{1}$
}

Received: 5 January 2020 / Accepted: 5 January 2020 / Published online: 21 January 2020

(c) The Author(s), under exclusive licence to The Japan Society of Human Genetics 2020

The Journal of Human Genetics published 155 papers in 2019. We prepared a special issue describing "Long-read sequencing in human genetics" in 2019, which was published in January 2020. I would like to express my sincere appreciation to the guest editor, Professor Yutaka Suzuki, and all the contributors for their great work. I hope the special issue will attract attention to a broad readership.

In 2020, we are planning a special issue dealing with new trends in GWAS. I look forward to it and also to receiving more exciting manuscripts for the Journal of Human Genetics from all over the world.

$\begin{array}{ll}\text { Catherine M Abbott } & \text { Gyaneshwer Chaubey } \\ \text { Pankaj B Agrawal } & \text { Takeshi Chiyomaru } \\ \text { Kiwamu Akagi } & \text { Kazuhiko Cho } \\ \text { Masato Akiyama } & \text { Sofie Lindgren Christiansen } \\ \text { Nana Akiyama } & \text { Kathleen Claes } \\ \text { Yoshimitsu Akiyama } & \text { James Collins } \\ \text { Manir Ali } & \text { Gregory Costain } \\ \text { Wolfram Antonin } & \text { Caroline Costedoat } \\ \text { Yoko Aoki } & \text { Fulvio Cruciani } \\ \text { Reiko Arakawa } & \text { Ranajit Das } \\ \text { Takuro Arimura } & \text { Peter de Knijff } \\ \text { Midori Awazu } & \text { Silvia de Rubeis } \\ \text { Shabeesh Balan } & \text { Matthew Deardorff } \\ \text { Carmen Birchmeier } & \text { Robert J Desnick } \\ \text { Craig Blackstone } & \text { Jie Ding } \\ \text { Francesco Brancati } & \text { David Duffy } \\ \text { Matthew E R butchbach } & \text { Yusuke Ebana } \\ \text { Francesc Calafell } & \text { Toshiaki Endo } \\ \text { Rosalba Carrozzo } & \text { Hezhi Fang } \\ \text { Claudia Carvalho } & \text { Mahmoud Fassad } \\ \text { Wei-Chiao Chang } & \text { Marcondes França junior }\end{array}$

Naomichi Matsumoto naomat@yokohama-cu.ac.jp

1 Department of Human Genetics, Graduate School of Medicine, Yokohama City University, Yokohama, Japan
Acknowledgements The Editor-in-Chief, on behalf of the Journal of Human Genetics Editorial Board and the Japan Society of Human Genetics, gratefully acknowledge the generous support from peer reviewers in carefully evaluating manuscripts under consideration. The following individuals provided reviews of manuscripts submitted to the Journal of Human Genetics in 2019:

\author{
Katsumi Higaki \\ Keiko Hikino \\ Shinjiro Hino \\ Naomi Hino-Fukuyo \\ Nattiya Hirankarn \\ Arisa Hirano \\ Masahiro Hiratsuka \\ Kenji Hirayama \\ Yuki Hitomi \\ Hitoshi Hiura \\ Momoko Horikoshi \\ Yoshihiro Hotta \\ Yiping Hou \\ Dirk Hubmacher \\ Fukiko Ichida \\ Daisuke Ichikawa \\ Hiroaki Ida \\ Masahito Ikawa \\ Masashi Ikeda \\ Yoshio Ikeda \\ Kazuo Imagawa \\ Minako Imamura \\ Tadashi Imanishi \\ Issei Imoto \\ Jun Inoue \\ Mika Ishige \\ Takayuki Ishige \\ Tetsuya Ishii
}


Kotaro Ishikawa

Taisuke Ishikawa

Hiroyuki Ishiura

Kaoru Ito

Nobuaki Ito

Takashi Ito

Tetsuya Ito

Motoki Iwasaki

Jennifer Johnston

Masayo Kagami

Semra Kahraman

Mayumi Kamada

Kengo Kamatani

Tadashi Kaname

Nobuo Kanazawa

Piranit Nik Kantaputra

Kenichi Kashimada

Kohji Kato

Takema Kato

Fumiki Katsuoka

Toshitaka Kawarai

Aya Kawasaki

Markus Keller

Raymond Kim

Tatsuya Kishino

Shin-ichiro Kitajiri

Hiroshi Kitoh

Karl Martin Klein

Kazuhiro Kobayashi

Masahisa Kobayashi

Yuta Kochi

Shuhei Komatsu

Eric Konnick

Uwe Kornak

Tomoki Kosho

Motomichi Kosuga

Shingo Koyama

Akiharu Kubo

Akatsuki Kubota

Pradeep G Kumar

Shigeo Kure

Kenji Kurosawa

Akira Kurozumi

Wibhu Kutanan

Kerstin Kutsche

Dan Larhammar

Lance Lee

Tomoko Lee

Marie Legendre

Hui Li

Mingkun Li

Yu-Chun Li

Zheng LI
Fan Liu

Siew-Kee Low

David Lynch

Artem Lysenko

Shiro Maeda

Wojciech Makalowski

Yoshio Makita

Boris A Malyarchuk

Julian Martinez-Agosto

Frith Matin

Keiko Matsubara

Nagahide Matsubara

Yoichi Matsubara

Shinya Matsuura

Tohru Matsuura

Johannes A Mayr

Jeremy McRae

Miriam H. Meisler

Niccolo E Mencacci

Daiki Miki

Jeffrey Hal Miner

Hiroyuki Mishima

Satomi Mitsuhashi

Norisato Mitsutake

Fuyuki Miya

Hiroko Miyadera

Taku Miyagawa

Hidehiko Miyake

Masahiro Miyake

Noriko Miyake

Takeshi Miyamoto

Satoko Miyatake

Seiichi Mori

Naoya Morisada

Shinichi Morishita

Hiroyuki Morita

Stefan Mundlos

Masaaki Muramatsu

Kei Murayama

Taisei Mushiroda

Keisuke Nagasaki

Yuki Nagata

Izumi Naka

Kazuhiko Nakabayashi

Masanori Nakagawa

Kenta Nakai

Katsunori Nakamura

Kimitoshi Nakamura

Akira Nakanishi

Hiroshi Nakanishi

Koichi Nakanishi

Yoshiharu Nakaoka

Mitsuko Nakashima
Kaname Nakatani

Akihiko Nakaya

Tomohiro Nakayama

Eiji Nanba

Hiroto Narimatsu

Akira Narita

Aya Narita

Taeko Naruse

Nobutoshi Nawa

Nao Nishida

Jo Nishino

Shinya Nishio

Daisuke Nishizawa

Emiko Noguchi

Eisei Noiri

Michael Nothnagel

Kandai Nozu

Chikahiko Numakura

Daniel Ocampo Daza

Katsuhisa Ogata

Tsutomu Ogata

Takehiko Ogawa

Tomoo Ogi

Osamu Ohara

Reiko Ohara

Jun Ohashi

Toya Ohashi

Kazutaka Ohi

Hidenori Ohnishi

Tohru Ohta

Nobuhiko Okamoto

Yasushi Okazaki

Masaya Oki

Torayuki Okuyama

Yosuke Omae

Heymut Omran

Yoshihiro Onouchi

Hiroki Oota

Takahiro Otani

Takanobu Otomo

Kouichi Ozaki

Takeshi Ozeki

Keiichi Ozono

Emma Palmer

Davut Pehlivan

Min-Sheng Peng

Paolo Peterlongo

Shubha R Phadke

Chris Phillips

Nicola Pirastu

Amélie Piton

Eliana Marisa Ramos

Kitiwan Rojnueangnit
Jill A Rosenfeld

Owen Ross

Edward A. Ruiz-Narváez

Lucky Ronald Runtuwene

Toshio Saito

Yoshiaki Saito

Shinji Saitoh

Naruya Saitou

Hirotomo Saitsu

Norio Sakai

Akihiro Sakurai

Ken Sakurai

Antonio Salas

Osamu Samura

Fabricio R Santos

Aiko Sasaki

Masayuki Sasaki

Takashi Sasaki

Suguru Sato

Akira Sawa

Hideaki Sawai

Daniel Francois Schorderet

Masahide Seki

Naohiko Seki

Donald Sellitti

Toshiyuki Seto

Qiuying Sha

Victoria Shabardina

Swarkar Sharma

A. Eliot Shearer

Qinghua Shi

Seiji Shibata

Daichi Shigemizu

Mihoko Shimada

Haruo Shimazaki

Minobu Shimazu

Kenji Shimizu

Norikazu Shimizu

Nobuyuki Shimozawa

Kouya Shiraishi

Yuichi Shiraishi

Curt Sigmund

David Sillence

Anu Sironen

Katie Snape

Hidenobu Soejima

Kyuyoung Song

Lea Starita

Zornitza Stark

Lois J Starr

Charlotte J Summer

Kai Sun

Liangdan Sun 
Supharat Suvichapanich

Ayako Suzuki

Hiromu Suzuki

Yoichi Suzuki

Zoltán Szabolcsi

Yasuharu Tabara

Hayato Tada

Atsushi Tajima

Go Tajima

Toshihiro Tajima

Atsushi Takahashi

Yuji Takahashi

Kyoko Takano

Kyoko Takashima

Atsushi Takata

Ryo Takata

Fumihiko Takeuchi

Kazuhiko Takeuchi
Yoshihisa Takiyama

Tiong Yang Tan

Zhiping Tan

Koichi Tanaka

Masayuki Tanemoto

Kohei Taniguchi

Chizu Tanikawa

Chikashi Terao

Henning Tiemeier

Katsushi Tokunaga

Akihito Tsubota

Katsuya Tsuchihara

Clare Turnbull

Mitsuharu Ueda

Yasutaka Ueda

Mitsugu Uematsu

Motoko Unoki

Shin-Ichi Usami
Joanne J van der Vis

Joris R Vermeesch

Barbara Vona

Takahito Wada

Chuan-Chao Wang

Matthew L Warman

Atsushi Watanabe

Ryo Watanabe

Grzegorz Wegrzyn

D Bradley Welling

Carol A. Wise

Jing Hao Wong

Philip Woodman

Chen Chi Wu

Angli Xue

Junya Yamagishi

Kiyoshi Yamaguchi

Kayono Yamamoto
Toshiyuki Yamamoto

Riu Yamashita

Keiko Yamazaki

Kazuki Yamazawa

Hsin-Chou Yang

Michio Yasunami

Sana Yokoi

Makoto Yoneda

Masayuki Yoshida

Reiko Yoshida

Takeshi Yoshida

Tomokatsu Yoshida

Koh-ichiro Yoshiura

Kei Yura

Massimo Zeviani

Zhongjun Zhou

Irena Zupanič Pajnič 